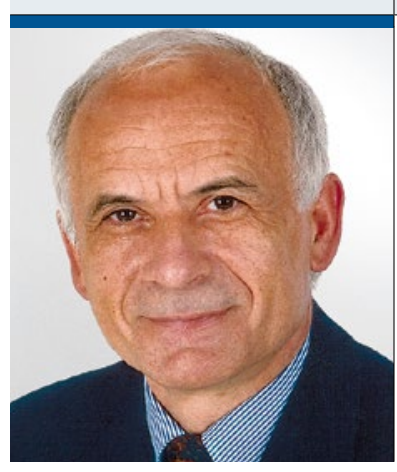

\section{Alfred Wirth}

Beirat und Leitlinienbeauftragter der Deutschen Adipositas Gesellschaft, Bad Rothenfelde

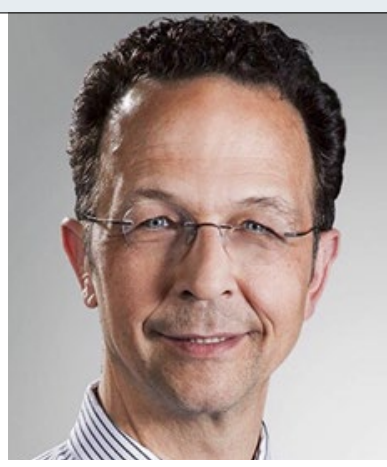

Thorsten Siegmund

Klinik für

Endokrinologie,

Diabtologie und

Angiologie, Klinikum Bogenhausen, München

\title{
Wichtige Aspekte bei Adipositas und neue Optionen für Diabetiker
}

\section{Herzfunktion und Krebs - wer denkt dabei schon an die Adipositas}

Immer noch wird die Adipositas als ein Symptom oder eine harmlose Variante einer Gesundheitsstörung betrachtet. Die Alltagswahrnehmung kann täuschen: Fast alle Folgekrankheiten der Adipositas sind nicht unmittelbar erkennbar und nicht wenige adipöse Mediziner tun sich schwer, die Adipositas vorurteilsfrei als Krankheit zu akzeptieren.

Bereits 1983 konnte in der Framingham-Studie gezeigt werden, dass eine Herzinsuffizienz bei Adipösen doppelt so häufig vorkommt wie bei Normalgewichtigen. Verantwortlich dafür sind Komorbiditäten wie Hypertonie, Vorhofflimmern, KHK, obstruktive Schlafapnoe, Hypoventilation sowie die vermehrte Fettmasse sui generis. Inzwischen sind auch die zugrunde liegenden Mechanismen weitgehend bekannt. Die Diagnose ist aufgrund der Fettleibigkeit erschwert. Das Leitsymptom der Herzinsuffizienz, die Dyspnoe, und eine die Adipositas häufig begleitende Veneninsuffizienz können zu einer Fehleinschätzung führen. Eine kombinierte klinische, laborchemische und apparative Diagnostik schafft jedoch Klarheit.

Wer aus gesundheitlichen Gründen das Gewicht halten oder reduzieren will, denkt selten an das adipositasassoziierte Krebsrisiko. Betroffen von einem erhöhten Krebsrisiko aufgrund eines erhöhten Gewichts sind viele Organe. Wenngleich das relative $\mathrm{Ri}$ siko bei Adipösen im Vergleich zu Normalgewichtigen bei manchen Krebsarten nur gering erhöht ist, ist der Anteil der Adipositas an allen Krebskrankheiten mit 5-10\% erheblich. Bleibt zu hoffen, dass zukünftig medizinische Fachpersonen auch auf Krebsaspekte hinweisen, wenn es um die Gesundheit geht.

\section{Lipid- und Glukosestoffwechsel optimieren}

Über die letzten 20 Jahre konnte die Langzeitprognose von Diabetikern u. a. durch einen konsequen- teren Einsatz von Statinen verbessert werden, deren Benefit für die Primär- und Sekundärprävention der KHK auch bei Diabetikern belegt ist. Dieser Effekt der Statine ist seit Publikation der 4S-Studie im Jahr 1994 bekannt. Durch weitere Studien hat sich der Zielwert für das LDL-Cholesterin kontinuierlich auf $<70 \mathrm{mg} / \mathrm{dl}$ für Hochrisikopatienten verändert. Nach Jahren ohne relevante Erkenntnisse aus Endpunktsudien konnte nun für einen weiteren Lipidsenker, Ezetemib, ein moderater zusätzlicher kardiovaskulärer Nutzen gezeigt werden. In wie weit bei der Lipidtherapie durch die Substanzklasse der PCSK9-Inhibitoren ein neues Kapitel der Prognoseoptimierung aufgeschlagen wird, bleibt spannend; die Präsentation der ersten Endpunktstudie im Herbst 2016 muss abgewartet werden.

Im Bereich der Diabetestherapie waren die letzten Jahre durch das Thema „Individualisierung“ der Therapie geprägt, $d . h$. individuelle Therapieziele in Abhängigkeit von Alter, Diabetesdauer, Hypoglykämierisiko der Therapie sowie Begleiterkrankungen und anderen Faktoren. Die Präsentation der Daten der EMPA-REG-Outcome-Studie zum SGLT2Hemmer Empagliflozin im Herbst 2015 und die auf dem amerikanischen Diabeteskongress 2016 präsentierten Daten zum GLP1-Rezeptoragonisten Liraglutid führen dazu, dass nun durch „modernere“ Substanzen die bisherigen Leitlinien zur Diabetestherapie neu geschrieben werden müssen. Spannend ist auch, dass die so prognoserelevante Herzinsuffizienz bei Diabetikern nun mit in den Vordergrund rückt.

Dies ist erst der Anfang: In den kommenden 4 Jahren erwarten wir Daten zu weiteren 12 Endpunktstudien mit moderneren Substanzen in der Diabetestherapie, das dürfte spannend werden.

\section{Alfred Wirth}

Thorsten Siegmund 